\title{
One Kind Special Gauss Sums and their Mean Square Values
}

\author{
Jianhong Zhao ${ }^{1}$ and Jiejie Gao $\mathbb{D}^{2}$ \\ ${ }^{1}$ Department of Public Basic Courses, West Yunnan University of Applied Sciences, Dali, Yunnan, China \\ ${ }^{2}$ School of Mathematics, Northwest University, Xi'an, Shaanxi, China
}

Correspondence should be addressed to Jiejie Gao; gaojiejie@stumail.nwu.edu.cn

Received 5 May 2021; Accepted 20 June 2021; Published 9 July 2021

Academic Editor: Basil Papadopoulos

Copyright (C) 2021 Jianhong Zhao and Jiejie Gao. This is an open access article distributed under the Creative Commons Attribution License, which permits unrestricted use, distribution, and reproduction in any medium, provided the original work is properly cited.

In this paper, we introduce one kind special Gauss sums; then, using the elementary and analytic methods to study the mean value properties of these kind sums, we obtain several exact calculating formulae for them.

\section{Introduction}

Let $q$ and $k$ be two positive integers with $q \geq 3$. For any integer $a \geq 1$ and Dirichlet character $\chi \bmod q$, we write $R_{k}(a)=[\sqrt[k]{a}]$, where $[x]$ denotes the greatest integer less than or equal to $x$. Then, we define the summations $G(n, \chi, k ; q)$ and $H(n, \chi, k ; q)$ as

$$
\begin{aligned}
& G(n, \chi, k ; q)=\sum_{a=1}^{q^{k}-1} \chi\left(R_{k}(a)\right) e\left(\frac{n a}{q}\right) \\
& H(n, \chi, k ; q)=\sum_{a=1}^{q^{k}-1} \chi\left(R_{k}(a)\right) e\left(\frac{n\left(a-R_{k}^{k}(a)\right)}{q}\right),
\end{aligned}
$$

where $e(y)=e^{2 \pi i y}$ and $n$ is any integer.

It is clear that $G(n, \chi, k ; q)$ is a generalization of the classical Gauss sums. In fact, if $k=1$, then we have $R_{1}(a)=a$ and

$$
G(n, \chi, 1 ; q)=\sum_{a=1}^{q-1} \chi(a) e\left(\frac{n a}{q}\right)=G(n, \chi ; q) .
$$

That is, $G(n, \chi, 1 ; q)$ becomes the classical Gauss sums $G(n, \chi ; q)$. Gauss sums play a very important role in the study of analytic number theory, and many number theory problems are closely related to it, so some scholars have studied the properties of the classical Gauss sums and obtained many meaningful and interesting results; some of them can be found in [1-14]. For this reason, we think that it is necessary and meaningful to study the properties of $G(n, \chi, k ; q)$.

On the contrary, $H(n, \chi, 2 ; q)$ is related to Dirichlet $L$-function $L(1, \chi)$. In fact, if $p$ is an odd prime, $\chi(-1)=-1$ and $n=0$, then we have

$$
|H(0, \chi, 2 ; p)|=2 \cdot\left|\sum_{m=1}^{p-1} m \chi(m)\right|=\frac{2 \cdot p^{3 / 2}}{\pi} \cdot|L(1, \chi)| .
$$

Therefore, the study of the sums $G(n, \chi, n ; q)$ and $H(n, \chi, n ; q)$ has extensive theoretical significance and application values.

In this study, as an attempt in this direction, we first study the mean value properties of $G(n, \chi, k ; q)$ and $H(n, \chi, k ; q)$. We shall use the elementary and analytic methods to prove the following several results.

Theorem 1. Let $p$ be an odd prime. Then, for any integer $n$ with $(n, p)=1$, we have the identity

$$
\sum_{\chi \bmod p}\left|\sum_{a=1}^{p^{2}-1} \chi\left(R_{2}(a)\right) e\left(\frac{n a}{p}\right)\right|^{2}=\frac{p(p-1)}{2 \sin ^{2}(\pi n / p)}-(p-1) .
$$

Theorem 2. Let $p$ be an odd prime. Then, we have the identity 


$$
\frac{1}{p-1} \sum_{\chi \bmod p}\left|\sum_{a=1}^{p^{3}-1} \chi\left(R_{3}(a)\right) e\left(\frac{n a}{p}\right)\right|^{2}= \begin{cases}\frac{p-\sqrt{p} \cdot \cos (\pi \cdot \overline{2} n / p)}{2 \sin ^{2}(\pi n / p)}-1, & \text { if } p \equiv 1 \bmod 12, \\ \frac{p+\sqrt{p} \cdot \cos (\pi \cdot \overline{2} n / p)}{2 \sin ^{2}(\pi n / p)}-1, & \text { if } p \equiv 5 \bmod 12, \\ \frac{p-\sqrt{p} \cdot \sin (\pi \cdot \overline{2} n / p)}{2 \sin ^{2}(\pi n / p)}-1, & \text { if } p \equiv 7 \bmod 12, \\ \frac{p+\sqrt{p} \cdot \sin (\pi \cdot \overline{2} n / p)}{2 \sin ^{2}(\pi n / p)}-1, & \text { if } p \equiv 11 \bmod 12,\end{cases}
$$

where $\bar{a}$ denotes the solution of the congruence equation $a x \equiv 1 \bmod p$.
Theorem 3. Let $p$ be an odd prime. Then, for any nonprincipal character $\chi \bmod p$, we have the identities

$$
\begin{aligned}
& \sum_{n=1}^{p-1}\left|\sum_{a=1}^{p^{2}-1} \chi\left(R_{2}(a)\right) e\left(\frac{n\left(a-R_{2}^{2}(a)\right)}{p}\right)\right|^{2}=\frac{p\left(p^{2}-1\right)}{12} \\
& \sum_{n=1}^{p-1}\left|\sum_{a=1}^{p^{2}-1} \chi\left(R_{2}(a)\right) e\left(\frac{n\left(a-R_{2}^{2}(a)\right)}{p}\right)\right|^{4}=\frac{p^{2}\left(p^{2}+11\right)\left(p^{2}-1\right)}{720} \\
& \sum_{n=1}^{p-1}\left|\sum_{a=1}^{p^{2}-1} \chi\left(R_{2}(a)\right) e\left(\frac{n\left(a-R_{2}^{2}(a)\right)}{p}\right)\right|^{6}=\frac{p^{3}\left(2 p^{2}-11\right)\left(p^{2}+17\right)\left(p^{2}-1\right)}{60480} .
\end{aligned}
$$

From Theorems 1 and 2, we can also deduce the following corollaries:

Corollary 1. Let $p$ be an odd prime. Then, we have the asymptotic formula

$$
\sum_{\chi \bmod p}\left|\sum_{a=1}^{p^{2}-1} \chi\left(R_{2}(a)\right) e\left(\frac{a}{p}\right)\right|^{2}=\frac{p^{4}}{2 \cdot \pi^{2}}+O\left(p^{3}\right) .
$$

Corollary 2. Let $p$ be an odd prime. Then, we have the asymptotic formula

$$
\sum_{\chi \bmod p}\left|\sum_{a=1}^{p^{2}-1} \chi\left(R_{3}(a)\right) e\left(\frac{a}{p}\right)\right|^{2}=\frac{p^{4}}{2 \cdot \pi^{2}}+O\left(p^{7 / 2}\right) .
$$

Notes. In fact, for any integer $h \geq 4$, using our methods and the results in [15], we can give an exact calculating formula for the general $2 \mathrm{hth}$ power mean:

$$
\sum_{n=1}^{p-1}\left|\sum_{a=1}^{p^{2}-1} \chi\left(R_{2}(a)\right) e\left(\frac{n\left(a-R_{2}^{2}(a)\right)}{p}\right)\right|^{2 h} .
$$

However, when $h$ is large, the calculation is more complicated, so we do not consider it.

\section{Several Lemmas}

This section, we need to give some simple lemmas, which are necessary in the proofs of our theorems. Of course, the proofs of these lemmas also need some knowledge of elementary and analytic number theory, in particular, the properties of the character sums and the classical Gauss sums modulo $p$. All these can be found in $[16,17]$, we do not repeat them. First, we have the following.

Lemma 1. Let $p$ be an odd prime and $k \geq 2$ be a fixed integer. Then, for any nonprincipal Dirichlet character $\chi \bmod p$, we have the identity 


$$
\begin{aligned}
\sum_{a=1}^{p^{k}-1} \chi\left(R_{k}(a)\right) e\left(\frac{n a}{p}\right) & =\sum_{m=1}^{p-1} \chi(m) \cdot \frac{e\left(n(m+1)^{k} / p\right)-e\left(n m^{k} / p\right)}{e(n / p)-1}, \\
\sum_{a=1}^{p^{k}-1} \chi\left(R_{k}(a)\right) e\left(\frac{n\left(a-R_{k}^{k}(a)\right)}{p}\right) & =\sum_{m=1}^{p-1} \chi(m) \cdot \frac{e\left(n\left((m+1)^{k}-m^{k}\right) / p\right)-1}{e(n / p)-1}, \\
\sum_{a=1}^{p^{2}-1} \chi\left(R_{2}(a)\right) e\left(\frac{n\left(a-R_{2}^{2}(a)\right)}{p}\right) & =\frac{\bar{\chi}(2 n) e(n / p) \tau(\chi)}{e(n / p)-1}
\end{aligned}
$$

where $\tau(\chi)=\sum_{a=1}^{p-1} \chi(a) e(a / p)$ denotes the classical Gauss sums.

Proof. First, we prove the first formula. Similarly, we can deduce the second one. For any positive integer $a$, it is clear that there are two integers $m \geq 1$ and $0 \leq i \leq(m+1)^{k}-m^{k}-$
1 such that $a=m^{k}+i$. This number pair $(m, i)$ not only exists, but it is unique. In fact, we have $m=[\sqrt[k]{a}]$ and $0 \leq i=a-[\sqrt[k]{a}]^{k} \leq(m+1)^{k}-1-m^{k}$, where $[x]$ denotes the greatest integer $\leq x$. From these results and the properties of geometric series, we have

$$
\begin{aligned}
\sum_{a=1}^{p^{k}-1} \chi\left(R_{k}(a)\right) e\left(\frac{n a}{p}\right) & =\sum_{m=1}^{p-1} \sum_{i=0}^{(m+1)^{k}-m^{k}-1} \chi(m) e\left(\frac{n\left(m^{k}+i\right)}{p}\right) \\
& =\sum_{m=1}^{p-1} \chi(m) e\left(\frac{n m^{k}}{p}\right) \frac{(m+1)^{k}-m^{k}-1}{\sum_{i=0}\left(\frac{n i}{p}\right)} \\
& =\sum_{m=1}^{p-1} \chi(m) e\left(\frac{n m^{k}}{p}\right) \frac{e\left(n\left((m+1)^{k}-m^{k}\right) / p\right)-1}{e(n / p)-1} \\
& =\sum_{m=1}^{p-1} \chi(m) \frac{e\left(n(m+1)^{k} / p\right)-e\left(n m^{k} / p\right)}{e(n / p)-1} .
\end{aligned}
$$

Now, we prove the third one. Taking $k=2$ in the second formula, note that $(m+1)^{2}-m^{2}=2 m+1$ and $\sum_{m=1}^{p-1} \chi(m)=0$, from the properties of the classical Gauss sums, and we have

$$
\begin{aligned}
\sum_{a=1}^{p^{2}-1} \chi\left(R_{2}(a)\right) e\left(\frac{n\left(a-R_{2}^{2}(a)\right)}{p}\right) & =\sum_{m=1}^{p-1} \chi(m) \cdot \frac{e(n(2 m+1) / p)-1}{e(n / p)-1} \\
& =\frac{1}{e(n / p)-1}\left[e\left(\frac{n}{p}\right) \sum_{m=1}^{p-1} \chi(m) e\left(\frac{2 n m}{p}\right)-\sum_{m=1}^{p-1} \chi(m)\right]=\frac{\bar{\chi}(2 n) e(n / p) \tau(\chi)}{e(n / p)-1}
\end{aligned}
$$


This proves Lemma 1.

Lemma 2. Let $p$ be an odd prime. Then, we have the identity

$$
\begin{aligned}
& \sum_{m=1}^{p-1}\left|\frac{e\left((m+1)^{3} / p\right)-e\left(m^{3} / p\right)}{e(1 / p)-1}\right|^{2} \\
& =\frac{2 p-\chi_{2}(3) \cdot e(\overline{4} / p) \cdot \tau\left(\chi_{2}\right)-\chi_{2}(-3) \cdot e(-\overline{4} / p) \cdot \tau\left(\chi_{2}\right)}{4 \sin ^{2}(\pi / p)}-1, \\
& \sum_{m=1}^{p-1}\left|\frac{e\left((m+1)^{2} / p\right)-e\left(m^{2} / p\right)}{e(1 / p)-1}\right|^{2}=\frac{p}{2 \sin ^{2}(\pi / p)}-1 .
\end{aligned}
$$

Proof. First, for any integer $n$ with $(n, p)=1$, from the properties of the quadratic Gauss sums, we have

$$
\sum_{m=0}^{p-1} e\left(\frac{n m^{2}}{p}\right)=1+\sum_{m=1}^{p-1}\left(1+\chi_{2}(m)\right) e\left(\frac{n m}{p}\right)=\left(\frac{n}{p}\right) \cdot \tau\left(\chi_{2}\right),
$$

where $\tau^{2}\left(\chi_{2}\right)=\chi_{2}(-1) \cdot p$ and $\chi_{2}=(* / p)$ denotes Legendre's symbol modulo $p$.

From (14) and the properties of the complete residue system modulo $p$, we have

$$
\begin{aligned}
& \sum_{m=1}^{p-1} e\left(\frac{3 m^{2}+3 m+1}{p}\right)=-e\left(\frac{1}{p}\right)+\sum_{m=0}^{p-1} e\left(\frac{3 m^{2}+3 m+1}{p}\right) \\
& =-e\left(\frac{1}{p}\right)+\sum_{m=0}^{p-1} e\left(\frac{3 \cdot \overline{4}(2 m+1)^{2}+\overline{4}}{p}\right)=-e\left(\frac{1}{p}\right)+\sum_{m=0}^{p-1} e\left(\frac{3 \cdot \overline{4} \cdot m^{2}+\overline{4}}{p}\right) \\
& =-e\left(\frac{1}{p}\right)+\chi_{2}(3 \cdot \overline{4}) \cdot e\left(\frac{\overline{4}}{p}\right) \cdot \tau\left(\chi_{2}\right)=-e\left(\frac{1}{p}\right)+\chi_{2}(3) \cdot e\left(\frac{\overline{4}}{p}\right) \cdot \tau\left(\chi_{2}\right) .
\end{aligned}
$$

Applying formula (15), we may immediately deduce

$$
\begin{aligned}
& \sum_{m=1}^{p-1}\left|\frac{e\left((m+1)^{3} / p\right)-e\left(m^{3} / p\right)}{e(1 / p)-1}\right|^{2}=\sum_{m=1}^{p-1}\left|\frac{e\left(\left(3 m^{2}+3 m+1\right) / p\right)-1}{e(1 / p)-1}\right|^{2} \\
& =\frac{1}{4 \sin ^{2}(\pi / p)} \cdot \sum_{m=1}^{p-1}\left(2-e\left(\frac{3 m^{2}+3 m+1}{p}\right)-e\left(\frac{-\left(3 m^{2}+3 m+1\right)}{p}\right)\right) \\
& =\frac{1}{4 \sin ^{2}(\pi / p)} \cdot\left[2 p-2-\sum_{m=1}^{p-1} e\left(\frac{3 m^{2}+3 m+1}{p}\right)-\sum_{m=1}^{p-1} e\left(\frac{-\left(3 m^{2}+3 m+1\right)}{p}\right)\right] \\
& =\frac{2 p-2+e(1 / p)+e(-1 / p)-\chi_{2}(3) \cdot e(\overline{4} / p) \cdot \tau\left(\chi_{2}\right)-\chi_{2}(-3) \cdot e(-\overline{4} / p) \cdot \tau\left(\chi_{2}\right)}{4 \sin ^{2}(\pi / p)} \\
& =\frac{2 p-\chi_{2}(3) \cdot e(\overline{4} / p) \cdot \tau\left(\chi_{2}\right)-\chi_{2}(-3) \cdot e(-\overline{4} / p) \cdot \tau\left(\chi_{2}\right)}{4 \sin ^{2}(\pi / p)}-1 .
\end{aligned}
$$


This proves the first formula in Lemma 2.

and we also have

Similarly, note that the identity

$$
\sum_{m=0}^{p-1} e\left(\frac{n m}{p}\right)= \begin{cases}p, & \text { if }(n, p)=p \\ 0, & \text { if }(n, p)=1,\end{cases}
$$

$$
\begin{aligned}
& \sum_{m=1}^{p-1}\left|\frac{e\left((m+1)^{2} / p\right)-e\left(m^{2} / p\right)}{e(1 / p)-1}\right|^{2}=\sum_{m=1}^{p-1}\left|\frac{e(2 m+1 / p)-1}{e(1 / p)-1}\right|^{2} \\
& =\frac{1}{4 \sin ^{2}(\pi / p)} \cdot \sum_{m=1}^{p-1}\left(2-e\left(\frac{2 m+1}{p}\right)-e\left(\frac{-2 m-1)}{p}\right)\right) \\
& =\frac{1}{4 \sin ^{2}(\pi / p)} \cdot\left[2 p-2-\sum_{m=1}^{p-1} e\left(\frac{2 m+1}{p}\right)-\sum_{m=1}^{p-1} e\left(\frac{-2 m-1}{p}\right)\right] \\
& =\frac{1}{4 \sin ^{2}(\pi / p)} \cdot\left[2 p-2+e\left(\frac{1}{p}\right)+e\left(\frac{-1}{p}\right)\right]=\frac{p}{2 \sin ^{2}(\pi / p)}-1 .
\end{aligned}
$$

This proves Lemma 2.

Lemma 3. Let $q \geq 3$ be an integer. Then, we have the identities

$$
\begin{aligned}
& \sum_{a=1}^{q-1} \frac{1}{\sin ^{2}(\pi a / q)}=\frac{q^{2}-1}{3} \\
& \sum_{a=1}^{q-1} \frac{1}{\sin ^{4}(\pi a / q)}=\frac{\left(q^{2}+11\right)\left(q^{2}-1\right)}{45} \\
& \sum_{a=1}^{q-1} \frac{1}{\sin ^{6}(\pi a / q)}=\frac{\left(2 q^{2}-11\right)\left(q^{2}+17\right)\left(q^{2}-1\right)}{945} .
\end{aligned}
$$

Proof. See [18] or Corollary 1 in [15].

\section{Proofs of the Theorems}

Applying three simple lemmas in Section 2, we can easily complete the proofs of our theorems. First, we prove Theorem 1. From the orthogonality of the characters modulo $p$ and Lemma 1 with $k=2$, we have

$$
\begin{aligned}
& \frac{1}{p-1} \sum_{\chi \bmod p}\left|\sum_{a=1}^{p^{2}-1} \chi\left(R_{2}(a)\right) e\left(\frac{n a}{p}\right)\right|^{2} \\
& =\frac{1}{4 \sin ^{2}(\pi n / p)} \cdot \sum_{m=1}^{p-1}\left|e\left(\frac{n(m+1)^{2}}{p}\right)-e\left(\frac{n m^{2}}{p}\right)\right|^{2}=\frac{p}{2 \sin ^{2}(\pi n / p)}-1 .
\end{aligned}
$$


This proves Theorem 1 .

Proof of Theorem 2. From the orthogonality of the characters modulo $p$ and Lemma 1 with $k=3$, we have

$$
\begin{aligned}
& \sum_{\chi \bmod p}\left|\sum_{a=1}^{p^{3}-1} \chi\left(R_{3}(a)\right) e\left(\frac{n a}{p}\right)\right|^{2} \\
& =\frac{p-1}{4 \sin ^{2}(\pi n / p)} \cdot \sum_{m=1}^{p-1}\left|e\left(\frac{n(m+1)^{3}}{p}\right)-e\left(\frac{n m^{3}}{p}\right)\right|^{2} \\
& =(p-1) \cdot\left[\frac{2 p-\chi_{2}(3) \cdot e(\overline{4} n / p) \cdot \tau\left(\chi_{2}\right)-\chi_{2}(-3) \cdot e(-\overline{4} n / p) \cdot \tau\left(\chi_{2}\right)}{4 \sin ^{2}(\pi n / p)}-1\right] .
\end{aligned}
$$

If $p \equiv 1 \bmod 12$, then $\chi_{2}(3)=\chi_{2}(-1)=1 \quad$ and $\tau\left(\chi_{2}\right)=\sqrt{p}$. From (21), we have

$$
\begin{aligned}
& \sum_{\chi \bmod p}\left|\sum_{a=1}^{p^{3}-1} \chi\left(R_{3}(a)\right) e\left(\frac{n a}{p}\right)\right|^{2} \\
& =\frac{(p-1)(p-\sqrt{p} \cdot \cos (\pi \cdot \overline{2} n / p))}{2 \sin ^{2}(\pi n / p)}-(p-1) .
\end{aligned}
$$

If $p \equiv 5 \bmod 12$, then $\chi_{2}(3)=-1, \quad \chi_{2}(-1)=1$, and $\tau\left(\chi_{2}\right)=\sqrt{p}$. From (21), we have

$$
\begin{aligned}
& \sum_{\chi \bmod p}\left|\sum_{a=1}^{p^{3}-1} \chi\left(R_{3}(a)\right) e\left(\frac{n a}{p}\right)\right|^{2} \\
& =\frac{(p-1)(p+\sqrt{p} \cdot \cos (\pi \cdot \overline{2} n / p))}{2 \sin ^{2}(\pi n / p)}-(p-1) .
\end{aligned}
$$

If $p \equiv 7 \bmod 12$, then $\chi_{2}(3)=-1, \chi_{2}(-1)=-1$, and $\tau\left(\chi_{2}\right)=i \cdot \sqrt{p}, i^{2}=-1$. From $(21)$, we have

$$
\begin{aligned}
& \sum_{\chi \bmod p}\left|\sum_{a=1}^{p^{3}-1} \chi\left(R_{3}(a)\right) e\left(\frac{n a}{p}\right)\right|^{2} \\
& =\frac{(p-1)(p-\sqrt{p} \cdot \sin (\pi \cdot \overline{2} n / p))}{2 \sin ^{2}(\pi n / p)}-(p-1) .
\end{aligned}
$$

If $p \equiv 11 \bmod 12$, then $\chi_{2}(3)=1, \chi_{2}(-1)=-1$, and $\tau\left(\chi_{2}\right)=i \cdot \sqrt{p}$. From $(21)$, we have

$$
\begin{aligned}
& \sum_{\chi \bmod p}\left|\sum_{a=1}^{p^{3}-1} \chi\left(R_{3}(a)\right) e\left(\frac{n a}{p}\right)\right|^{2} \\
& =\frac{(p-1)(p+\sqrt{p} \cdot \sin (\pi \cdot \overline{2} n / p))}{2 \sin ^{2}(\pi n / p)}-(p-1) .
\end{aligned}
$$

Now, Theorem 2 follows from (22), (23), (24), and (25).

Now, we prove Theorem 3. For any nonprincipal character modulo $p$, note that $|\tau(\chi)|=\sqrt{p}$; from the third formula in Lemma 1, we have $\left|\sum_{a=1}^{p^{2}-1} \chi\left(R_{2}(a)\right) e\left(\frac{n\left(a-R_{2}^{2}(a)\right)}{p}\right)\right|^{2}=\frac{p}{4 \sin ^{2}(\pi n / p)}$.

From (26) and Lemma 3, we have

$$
\begin{aligned}
& \sum_{n=1}^{p-1}\left|\sum_{a=1}^{p^{2}-1} \chi\left(R_{2}(a)\right) e\left(\frac{n\left(a-R_{2}^{2}(a)\right)}{p}\right)\right|^{2} \\
& =\sum_{n=1}^{p-1} \frac{p}{4 \sin ^{2}(\pi n / p)}=\frac{p\left(p^{2}-1\right)}{12} .
\end{aligned}
$$

Similarly, from (26) and Lemma 3, we also have

$$
\begin{aligned}
& \sum_{n=1}^{p-1}\left|\sum_{a=1}^{p^{2}-1} \chi\left(R_{2}(a)\right) e\left(\frac{n\left(a-R_{2}^{2}(a)\right)}{p}\right)\right|^{4} \\
& =\sum_{n=1}^{p-1} \frac{p^{2}}{16 \sin ^{4}(\pi n / p)}=\frac{p^{2}\left(p^{2}+11\right)\left(p^{2}-1\right)}{720}, \\
& \sum_{n=1}^{p-1}\left|\sum_{a=1}^{p^{2}-1} \chi\left(R_{2}(a)\right) e\left(\frac{n\left(a-R_{2}^{2}(a)\right)}{p}\right)\right|^{6} \\
& =\sum_{n=1}^{p-1} \frac{p^{3}}{64 \cdot \sin ^{6}(\pi n / p)} \\
& =\frac{p^{3}\left(2 p^{2}-11\right)\left(p^{2}+17\right)\left(p^{2}-1\right)}{60480} .
\end{aligned}
$$

Now, Theorem 3 follows from (27)- (29).

This completes the proofs of all our results.

\section{Data Availability}

No data were used to support this study.

\section{Conflicts of Interest}

The authors declare that there are no conflicts of interest regarding the publication of this paper. 


\section{Authors' Contributions}

All authors have equally contributed to this work. All authors read and approved the final manuscript.

\section{Acknowledgments}

This work was supported by the N. S .F. (11771351) of P. R. China and WYUAS (2021RCKY0002).

\section{References}

[1] L. Chen and J. Y. Hu, "A linear recurrence formula involving cubic Gauss sums and kloosterman sums," Acta Mathematica Sinica, Chinese Series, vol. 61, pp. 67-72, 2018.

[2] Z. Y. Chen and W. P. Zhang, "On the fourth-order linear recurrence formula related to classical gauss sums," Open Mathematics, vol. 15, pp. 1251-1255, 2017.

[3] L. Chen, "On classical gauss sums and some of their properties," Symmetry, vol. 10, no. 11, p. 625, 2018.

[4] Y. Y. Liu and W. P. Zhang, "The linear recurrence formula of the hybrid power mean involving the cubic gauss sums and two-term exponential sums," Journal of Shaanxi Normal University (Natural Science Edition), vol. 45, pp. 14-17, 2017.

[5] X. Wang, "A note on the fourth power mean of the two-term exponential sums," Acta Mathematica Sinica, Chinese Series, vol. 62, pp. 261-268, 2019.

[6] X. X. Lv and W. P. Zhang, "A new hybrid power mean involving the generalized quadratic gauss sums and sums analogous to kloosterman sums," Lithuanian Mathematical Journal, vol. 57, no. 3, pp. 359-366, 2017.

[7] X. X. Li and J. Y. Hu, "The hybrid power mean quartic gauss sums and kloosterman sums," Open Mathematics, vol. 15, pp. 151-156, 2017.

[8] D. Han, "A hybrid mean value involving two-term exponential sums and polynomial character sums," Czechoslovak Mathematical Journal, vol. 64, pp. 53-62, 2014.

[9] L. Chen and Z. Y. Chen, "Some new hybrid power mean formulae of trigonometric sums," Advances in Difference Equations, vol. 2020, no. 1, Article ID 220, 2020.

[10] S. M. Shen and W. P. Zhang, "On the quartic gauss sums and their recurrence property," Advances in Difference Equations, vol. 2017, no. 1, Article ID 220, 2017.

[11] S. Chern, "On the power mean of a sum analogous to the kloosterman sum," Bulletin Mathematique de la Societe des Science Mathematique de Roumanie, vol. 62, pp. 77-92, 2019.

[12] J. Greene and D. Stanton, "The triplication formula for gauss sums," Aequationes Mathematicae, vol. 30, no. 1, pp. 134-141, 1986.

[13] J. Z. Wang and Y. K. Ma, "The hybrid power mean of the $k$-th gauss sums and kloosterman sums," Journal of Shaanxi Normal University (Natural Science Edition), vol. 45, pp. 5-7, 2017.

[14] X. H. Wang and J. J. Wang, "On the power mean of $L$-functions weighted by the the $k$-th gauss sums," Journal of Shaanxi Normal University (Natural Science Edition), vol. 45, pp. 28-30, 2017.

[15] W. P. Zhang and D. Han, "On the chebyshev polynomials and some of their reciprocal sums," Symmetry, vol. 12, no. 5, p. 704, 2020.

[16] T. M. Apostol, Introduction to Analytic Number Theory, Springer-Verlag, New York, NY, USA, 1976.

[17] K. Ireland and M. Rosen, A Classical Introduction to Modern Number Theory, Springer-Verlag, New York, NY, USA, 1982.
[18] Y. K. Ma and X. X. Lv, "Some identities involving the reciprocal sums of one kind chebyshev polynomials," Mathematical Problems in Engineering, vol. 2017, Article ID 4194579, 5 pages, 2017. 